\title{
Exploiting nonlinearity in a flapping wing mechanism of a bio inspired micro air vehicle to enhance energy efficiency
}

\author{
Ali Abolfathi ${ }^{1, *}$ \\ ${ }^{1}$ Dept. of Mechanical Engineering, University College London (UCL), Roberts Engineering Building, London, UK WC1E 7JE
}

\begin{abstract}
In recent years, there was a great interest in developing flying drones with similar capabilities as flying insects. It is suggested that the flapping frequency of insects coincides with the resonance frequency of their flight mechanism to enhance the power consumptions. In this paper, the effect of nonlinearity in the flight mechanism on the power consumption is investigated. A simple nonlinear model of the insect flight mechanism is developed and normalised to study the effect of different parameters on its performance. Both bistable and hardening nonlinearity are considered. It is shown that for a harmonic loading, the bistable systems reach their peak power at lower frequencies when compared to the corresponding linear system. The maximum power factor of nonlinear oscillator would be lower than the liner one. It is also shown that the peak active power of the bistable system has a higher value than the linear system if the loading function is a pulse square signal.
\end{abstract}

\section{Introduction}

Flying insects have evolved over thousands of years and have become efficient flying machines. They served as a source of inspiration for mini and micro unmanned air vehicles [1,2]. According to Greenewalt [3], the frequency of flapping is constant at different flight regimes. The flying birds or insects are modelled as a damped linear oscillator which operates at its resonance frequency to minimise the energy consumption. Fischer et. al. [4] proposed mechanisms for flapping wing robots that operate at their resonance frequency to minimise the power consumption. Madangopal et. al. [5] used four bar mechanisms for a flapping drone and used linear springs to mimic the elastic energy storage of the insects. They optimised the geometrical parameters and spring constant to achieve the minimum energy consumption. Baek et. al. [6] designed a flapping wing drone and optimised its performance considering the effect of battery and electrical motor. Bronson et. al. [7] and Bao et. al. [8] developed flapping wings that can be excited at the resonance frequency of the wing.

Goosen et. al. [9] proposed a new design for a flapping wing drone. They listed nonlinearity as one of the difficulties in implementing a flying machine at its resonance frequency in practice. Ramananarivo et. al. [10] developed a nonlinear model of a beam like wing having cubic stiffness nonlinearity and quadratic damping term. They concluded that the resonance is not providing the maximum efficiency, but the temporal evolution of the wing is optimised to increase the aerodynamic efficiency.

In insects with indirect flight muscles, the flight muscles are not directly connected to the wings but they cause a deformation of the thorax [11]. The movement of the thorax transfers to the wings through a complex mechanism. Different models are suggested for the wingmuscle interface [12] but its exact function yet still to be explored. The common theme between different models is the nonlinearity in restoring force of the "flight mechanism". The benefit of such a nonlinear mechanism is attributed to an increase in the kinetic energy of the wings $[13,14]$, modulation of the flapping amplitude [15] and an increase in the flapping speed during half of the flapping cycle [16].

In this paper, the energy efficiency of a flight mechanism is investigated by examining the active and reactive powers of a nonlinear oscillatory system. The power that conduct useful work in a system is called active power and the power that is used to accelerate masses and deform elastic elements of the system is the reactive power [17]. In theory, the energy attributed to the reactive power returns to the source in a cycle. However, it increases the energy loss as most mechanical actuators do not have an energy recovery capability. A larger actuator is also required to compensate the reactive power requirement. In a linear oscillator at the resonance, the stiffness and inertia term cancel out and the reactive power become zero which is not the case in a nonlinear oscillator.

A lumped parameter model in form of a Duffing oscillator is used in this study as a simplified representation of a flapping wing aerial vehicle. Both bistable and monostable oscillator are considered. The generated lift is modelled by a linear damping term for the simplicity. The performance of the system for a range of parameters is evaluated and it is compared with a linear counterpart.

\footnotetext{
* Corresponding author: a.abolfathi@ucl.ac.uk
} 


\section{A nonlinear model}

A schematic view of the "click mechanism" which was proposed in Ref. [18] is shown in Fig. 1(a). A mechanical model of this mechanism is proposed by Brenan et. al. [13] and is shown in Fig. 1(b). The length $2 b$ is the distances between the supports when springs are unloaded. The linear horizontal springs model the stiffness of notum and mesopleural process. The mechanism will snap through the unstable static equilibrium position if a force applies downward at point C. There are two static stable equilibrium positions which are shown by solid and dashed line in Fig. 1(b). There is also an unstable equilibrium position where the levers are horizontal. Such a system is called bistable as there are two equilibrium points.

a)

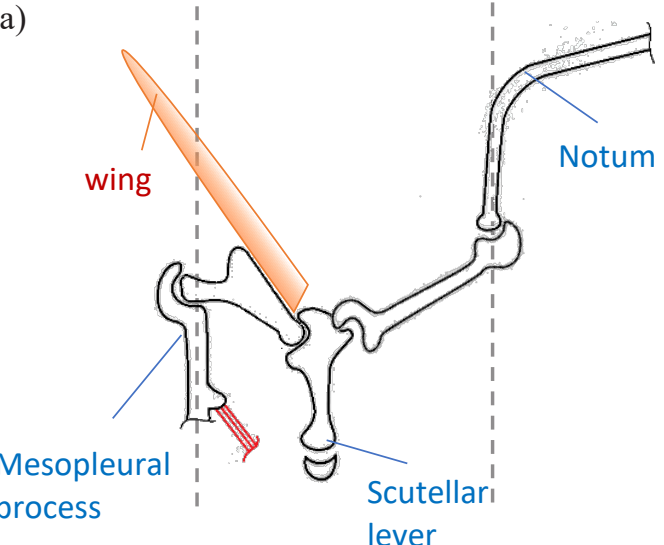

b)

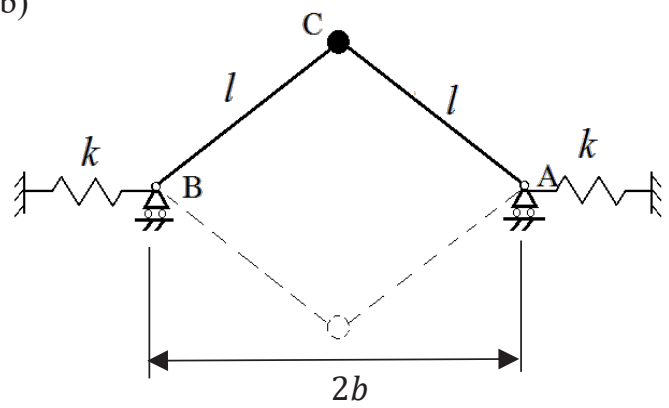

Fig. 1. a) A schematic view of insect click mechanism reproduced from Ref. [18]. b) Mechanical model of the click mechanism.

To account for the stiffness of scutellar lever (Fig. 1(a)) a vertical spring can be added to the mechanical model at point $\mathrm{C}$ in parallel to a damper as shown in Fig. 2. The damper is to model the aerodynamic load. The stiffness of the vertical spring can compensate the negative stiffness resulted from the lever mechanism and depending on the ratio between the vertical and horizontal springs $\alpha=k / k_{v}$ such a system can be bistable or only possess a single stable equilibrium position, hereafter will be referred to as monostable system. Different forcedeflection curves for different values of $\alpha$ are shown in Fig. 3 for a ratio of $b / l=0.7$.

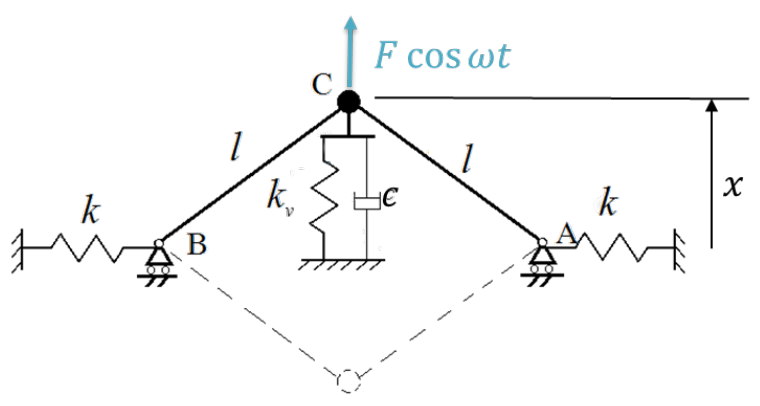

Fig. 2. Complete model of the insect flight mechanism.

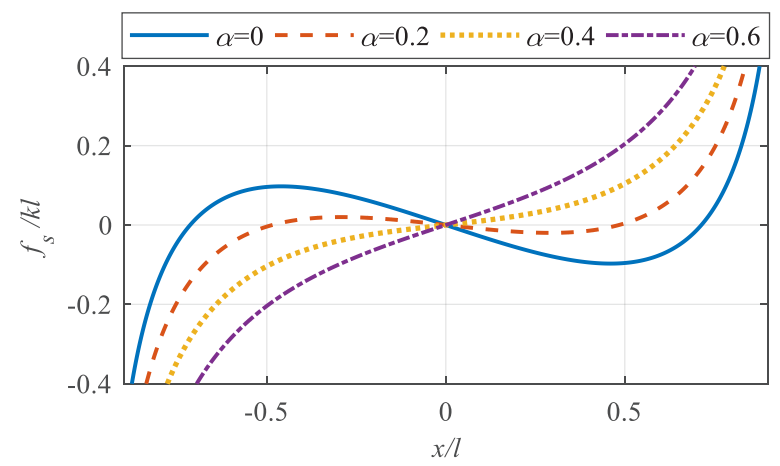

Fig. 3. Nondimensional force as a function of displacement of the flight mechanism model for different ratio of $\alpha$ and $b / l=$ 0.7 .

The equation of motion of the single degree of freedom model of the flapping mechanism can be obtained,

$$
m \ddot{x}+c \dot{x}+f_{s}(x)=F \cos \omega t
$$

where $f_{S}(x)$ is the nonlinear spring force. The spring reaction force can be approximated by a third order polynomial and the equation of motion would have the form of,

$$
m \ddot{x}+c \dot{x}+k_{1} x+k_{3} x^{3}=F \cos \omega t
$$

where $k_{1}$ and $k_{3}$ are stiffness coefficients and can be obtained as function of system parameters [13]. Depending on the chosen values for $\alpha$ and $\beta$, linear stiffness term $k_{1}$ can have a positive or a negative value. In this paper, it is tried to understand how flapping can be benefitted from nonlinearity. The equation of motion is normalised to make it possible to analyse the effect of different parameters on flapping performance,

$$
\hat{x}^{\prime \prime}+2 \zeta \hat{x}^{\prime}+\kappa \hat{x}+\gamma \hat{x}^{3}=\cos \Omega t
$$

where $k=\left|k_{1}\right|, \omega_{n}=\sqrt{k / m}, \quad \tau=\omega_{n} t, \quad \hat{x}=x / x_{0}$, $x_{0}=F / k, \quad \zeta=c /\left(2 m \omega_{n}\right), \quad \Omega=\omega / \omega_{n}, \quad \kappa=k_{1} / k$, $\gamma=x_{0}^{2} k_{3} / k_{1}$ and dash $(\boldsymbol{\square})^{\prime}$ represents differentiation with respect to non-dimensional time $\tau$. The normalised linear stiffness term $\kappa$ can have a value of 1 or -1 depending on the type of the system, i.e. bistable or monostable. The normalised nonlinear stiffness term $\gamma$ can be considered as the ratio between nonlinear spring 
force and linear spring force at the displacement $x_{0}$. It can also be linked to the applied force and thus is a function of the amplitude of response.

\section{Power}

The power that is used to produced aerodynamic lift is used as a measure of the performance here. This is called active or true power [17] and its average can be obtained over a period by calculating the work of the external force over a period,

$$
P=\frac{1}{T} \int_{0}^{T} F(t) \dot{x} d t
$$

where $F(t)$ is the applied force. The power can be nondimensionalised as $\hat{P}=P /\left(F x_{0} \omega_{n}\right)$. The active power is the result of work done on the damping element and the same result can be obtained if external force is substituted by the damping force in Eq. (4).

There is no net transfer of energy in a cycle through the work conducted on mass and stiffness elements of the system, and the part of the power that is required to move them is called reactive power. Its average can be obtained over a cycle similarly,

$$
Q=\frac{1}{T} \int_{0}^{T}\left|f_{s}(x(t))-m \ddot{x}\right| \dot{x} d t
$$

where $Q$ is the average reactive power and can be nondimensionalised as $\hat{Q}=Q /\left(F x_{0} \omega_{n}\right)$. It is important to note that although the net energy transfer due to reactive power is zero over a cycle, but it need to be considered in selection of the actuator as it should be sized based on the apparent power $S=\sqrt{P^{2}+Q^{2}}$. The ratio between active power and apparent power is called power ratio and is considered as a measure of the performance of the system in this study. A power ratio of one would result when all the power is used to conduct useful work.

\section{Harmonic loading}

The active and reactive powers are obtained in this section for a range of parameters to compare the performance of nonlinear and linear system in term of energy efficiency. A purely harmonic loading is assumed to be applied to the mechanism. The aerodynamic force is modelled through the damping force and as the purpose of the oscillator is to produce lift, it is expected that the damping coefficient be high. By comparing the ratio of the powers reported in Ref. [19], a damping ratio of 0.9 can be obtained for a linear oscillator.

\subsection{Bistable oscillator}

The nondimensional active and reactive power for the bistable mechanism is shown in Fig. 4 as a function of excitation frequency for three different value of $\gamma$. The frequency of the peak active power increases by increasing $\gamma$. The drop in active power immediately after the peak corresponds to the bifurcation where the interwell oscillation become chaotic before converting to intra-well oscillation. The peak in active power of the linear oscillator occurs at a normalised frequency of 1 where the reactive power is equal to zero. For a linear oscillator at the natural frequency, the inertia and stiffness forces cancel out and the input energy dissipates completely through damping term. However, for a nonlinear oscillator, the reactive power never become zero because multiple harmonics exist in the response.

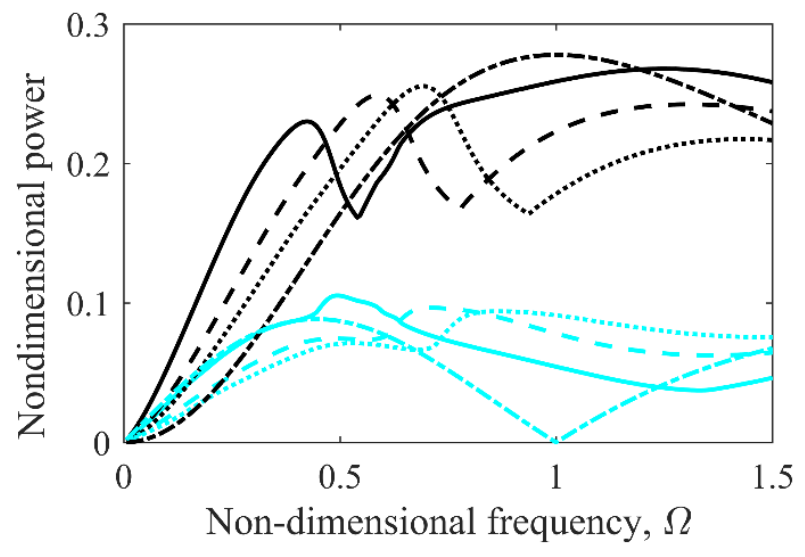

Fig. 4. Nondimensional power as a function of frequency for harmonic loading, $\kappa=-1$ and $\zeta=0.9$. black lines: active power $\hat{P}$, cyan lines: reactive power $\hat{Q}$. Solid line: $\gamma=1$; dashed line: $\gamma=2$; dotted line: $\gamma=3$; dashed-dotted line: linear oscillator.

Power factor is plotted in the Fig. 5 as a function of frequency for the bistable system and the linear oscillator. The power factor is equal to unity for the linear oscillator at the natural frequency of the system. The peak in power factor is lower for nonlinear oscillator and is about $\% 95$ but it is higher at low frequencies compared to the linear oscillator.

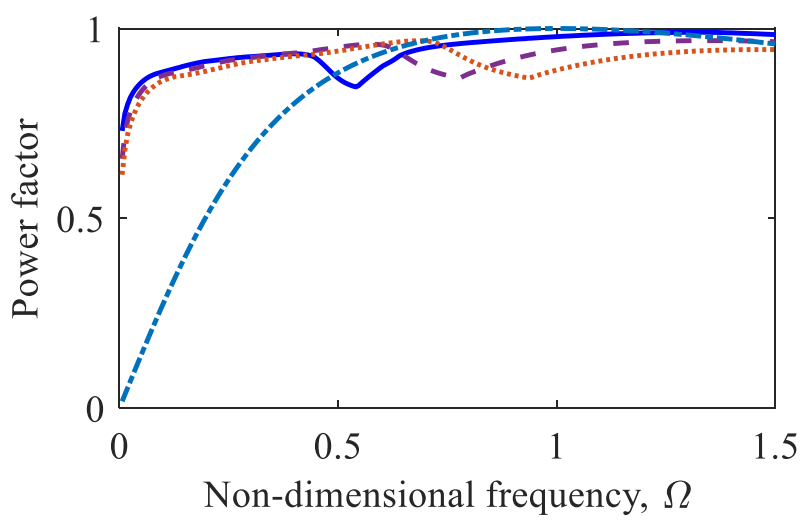

Fig. 5: Power factor as a function of frequency for harmonic loading, $\kappa=-1$ and $\zeta=0.9$. Solid line: $\gamma=1$; dashed line: $\gamma=2$; dotted line: $\gamma=3$; dashed-dotted line: linear oscillator.

Although the damping factor is expected to be high due the nature of the oscillatory system, its exact value is not known. To evaluate the effect of damping, the active power for three different level of damping is shown in Fig. 6 for the bistable oscillator with $\gamma=0.2$. The lower damping level cause an increase in the peak of the nondimensional active power as well as a shift in its 
frequency to a higher frequency. Reducing the damping also cause a slight reduction in the power factor which is not shown here for brevity.

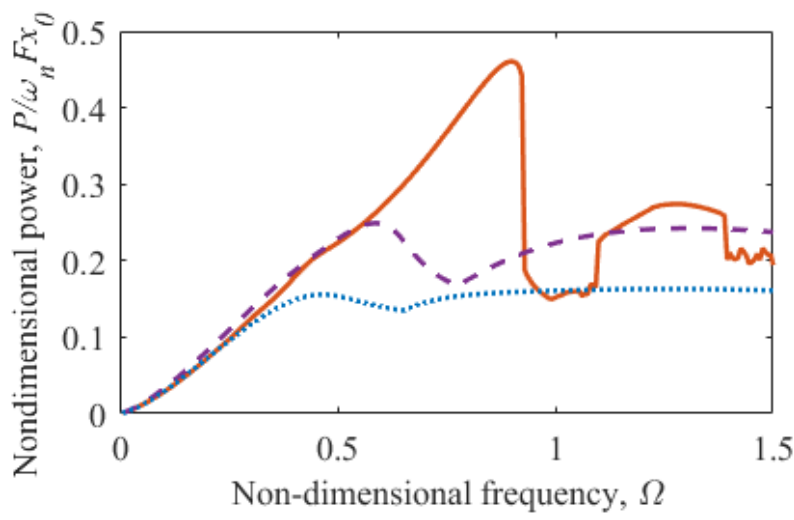

Fig. 6. Nondimensional power as a function of frequency for harmonic loading, $\kappa=-1$ and $\gamma=2$. Solid line: $\zeta=0.5$; dashed line: $\zeta=0.9$; dotted line: $\zeta=1.5$.

\subsection{Monostable oscillator}

The active and reactive power for a monostable oscillator $(\kappa=1)$ is shown in Fig. 7 for three different value of $\gamma$. Similar to Fig. 4, damping ratio is assumed to be equal to 0.9. The hardening nonlinearity of the oscillator cause a shift in the peak frequency to higher frequencies compared to the linear oscillator. Furthermore, the reactive power has a minimum almost equal to zero similar to the linear system. The resulted power factor is shown in Fig. 8. The nonlinear oscillator has a maximum power factor close to unity. The high damping ratio makes the effect of nonlinearity on the dynamics of the monostable oscillator minimal.

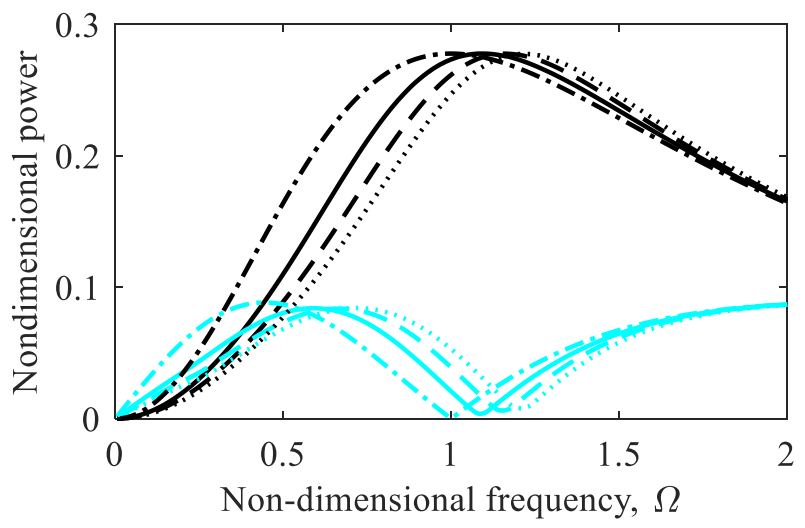

Fig. 7. Nondimensional power as a function of frequency for harmonic loading, $\kappa=1$ and $\zeta=0.9$. black lines: active power $\hat{P}$, cyan lines: reactive power $\hat{Q}$. Solid line: $\gamma=1$; dashed line: $\gamma=2$; dotted line: $\gamma=3$; dashed-dotted line: linear oscillator.

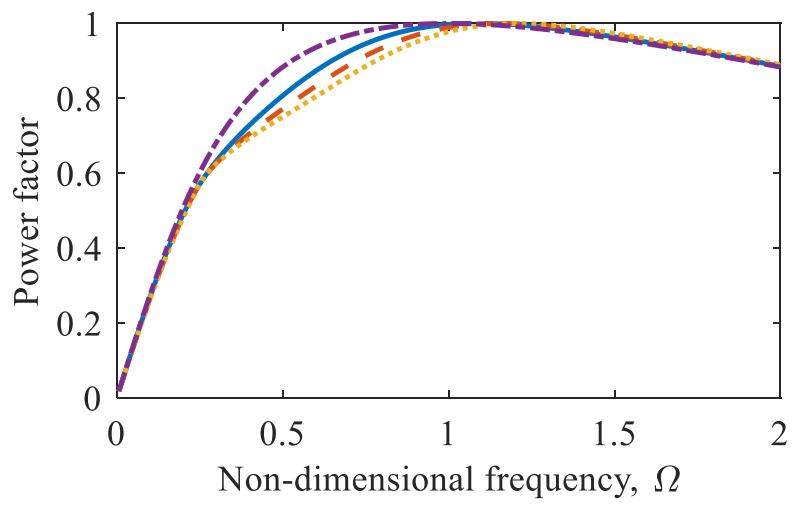

Fig. 8. Power factor as a function of frequency for $\kappa=1$ and $\zeta=0.9$. Solid line: $\gamma=1$; dashed line: $\gamma=2$; dotted line: $\gamma=$ 3 ; dashed-dotted line: linear oscillator.

\section{Non-harmonic loading}

The forcing function of insect muscles are not most likely a simple harmonic. The existence of higher harmonics in a non-sinusoidal loading function can be beneficial to the performance of a nonlinear oscillator by increasing its power factor.

\subsection{Bistable oscillator}

The active and reactive power for a bistable system when subjected to a square loading function is shown in Fig. 9. The amplitude of the peak power is almost constant for three different values of nonlinearity and is higher than the linear oscillator. The amplitude of peak active power is almost two times of that for the same oscillator when harmonically loaded (Fig. 4). The power factor is shown in Fig. 10. The power factor is very close to one in all frequencies due to existence of multiple harmonics in the forcing function.

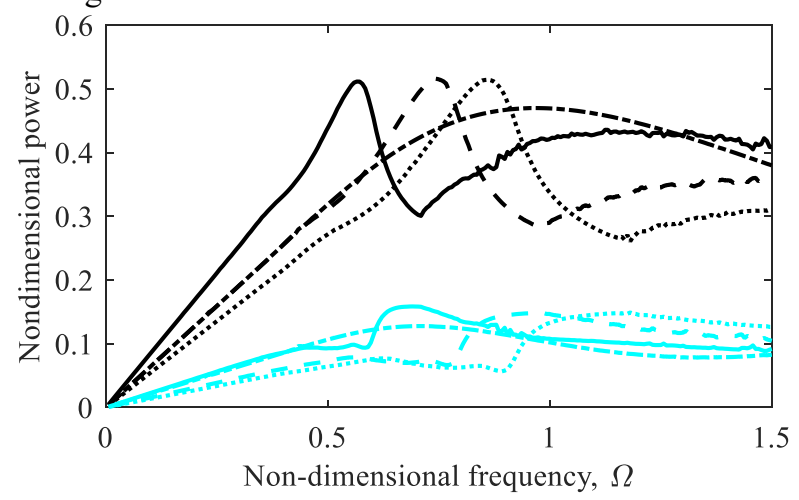

Fig. 9. Nondimensional power as a function of frequency for square loading, $\kappa=-1$ and $\zeta=0.9$. black lines: active power $\widehat{P}$, cyan lines: reactive power $\widehat{Q}$. Solid line: $\gamma=1$; dashed line: $\gamma=2$; dotted line: $\gamma=3$; dashed-dotted line: linear oscillator. 


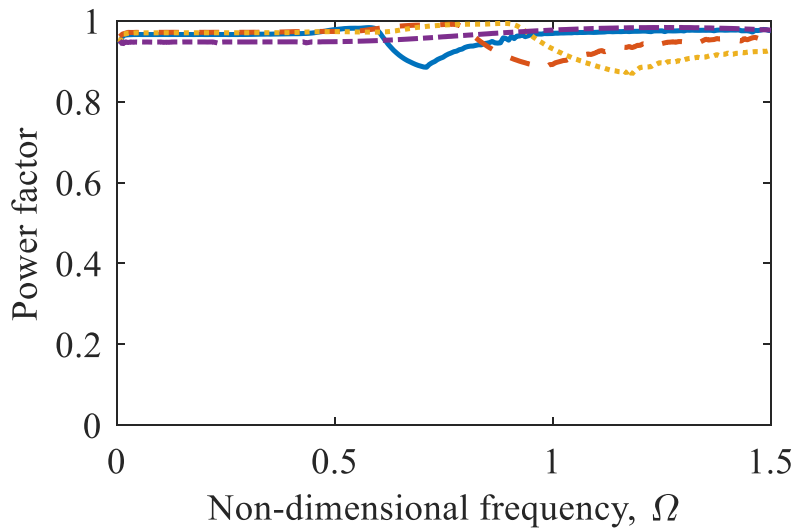

Fig. 10. Power factor as a function of frequency for square loading, $\kappa=-1$ and $\zeta=0.9$. Solid line: $\gamma=1$; dashed line: $\gamma=2$; dotted line: $\gamma=3$; dashed-dotted line: linear oscillator.

\subsection{Monostable oscillator}

The power curves of the monostable oscillator alongside a linear one are shown in Fig. 11 when subjected to a square pulse forcing function. The amplitude of active power is larger than the harmonic loading shown in Fig. 7. The hardening nonlinearity cause the peaks of active power to shift to higher frequencies. Similar to the bistable system, the power factor would be close to unity which is not shown here for brevity.

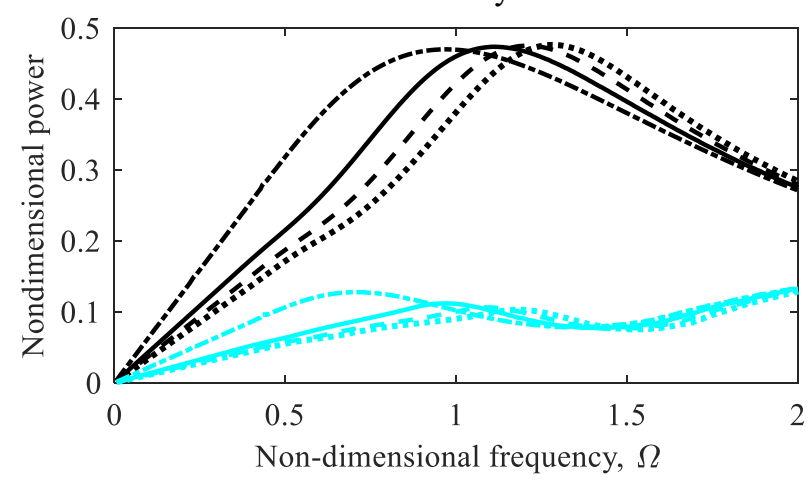

Fig. 11. Nondimensional power as a function of frequency for square loading, $\kappa=1$ and $\zeta=0.9$. Black lines: active power $\hat{P}$, cyan lines: reactive power $\hat{Q}$. Solid line: $\gamma=1$; dashed line: $\gamma=2$; dotted line: $\gamma=3$; dashed-dotted line: linear oscillator.

\section{Conclusions}

The nonlinearity in insect flight mechanism is modelled by a cubic nonlinearity. The mechanism can be bistable or monostable depending on the values of system parameters. The aerodynamic lift generation is modelled by a linear damper and the corresponding active power is obtained. The magnitude and the frequency at which the active power has its maximum value and the power factor are obtained in order to compare the effect of different parameters.

For a bistable mechanism, the active power has a peak value at a lower frequency than the natural frequency of a comparable linear oscillator. The power factor of a linear oscillator is unity at its natural frequency but for a nonlinear oscillator it has a value smaller than one. For a monostable system with hardening stiffness the peak power shifts to the higher frequencies. The effect of nonlinearity is minimum in this case as the damping ratio is high.

A square pulse forcing function is examined to assess a nonharmonic forcing function. The peak active power for bistable nonlinear system is higher than the linear one which implies a higher lift generation. Furthermore, the power factor has a value close to unity for the frequency range that is examined here.

\section{References}

1. D. Floreano and R.J. Wood, Nature 521 7553, 460-466 (2015).

2. G. Cai, J. Dias, and L. Seneviratne, Unmanned Syst. 2 2, 175-199 (2014).

3. C.H. Greenewalt, Proc. Am. Philos. Soc. 104 6, 605-611 (1960).

4. G. Fischer, A.G. Cox, M.A. Gogola, M.K. Gordon, N.O. Lobontiu, D.J. Monopoli, E. Garcia, and M. Goldfarb, Elastodynamic locomotion in mesoscale robotic insects, in: Ed. by Y. Bar-Cohen, Smart Struct. Mater. 1999 Electroact. Polym. Actuators Devices, (International Society for Optics and Photonics, Newport Beach, CA, US 1999), pp. 362-368.

5. R. Madangopal, Z.A. Khan, and S.K. Agrawal, J. Mech. Des. 127 4, 809 (2005).

6. S.S. Baek, K.Y. Ma, and R.S. Fearing, Efficient resonant drive of flapping-wing robots, in: 2009 IEEE/RSJ Int. Conf. Intell. Robot. Syst., (IEEE, St. Louis, MO, USA 2009), pp. 2854-2860.

7. J.R. Bronson, J.S. Pulskamp, R.G. Polcawich, C.M. Kroninger, and E.D. Wetzel, PZT MEMS Actuated Flapping Wings for Insect-Inspired Robotics, in: 2009 IEEE 22nd Int. Conf. Micro Electro Mech. Syst., (IEEE, Sorrento, Italy 2009), pp. 1047-1050.

8. X.Q. Bao, T. Dargent, S. Grondel, J.B. Paquet, and E. Cattan, Microelectron. Eng. 88 8, 22182224 (2011).

9. J.F.L. Goosen, H.J. Peters, Q. Wang, P. Tiso, and F. Van Keulen, Resonance Based Flapping Wing Micro Air Vehicle, in: Int. Micro Air Veh. Conf. Flight Compet., Toulouse, France 2013).

10. S. Ramananarivo, R. Godoy-Diana, and B. Thiria, Proc. Natl. Acad. Sci. U. S. A. 108 15, 5964-9 (2011).

11. R.F. (Reginald F. Chapman, S.J. Simpson, and A.E. (Angela E. Douglas, The insects : structure and function n.d.).

12. A.R. ENNOS, J. Exp. Biol. 127 1, (1987).

13. M.J. Brennan, S.J. Elliott, P. Bonello, and J.F. V Vincent, J. Theor. Biol. 224 2, 205-213 (2003).

14. B. Tang and M.J. Brennan, J. Theor. Biol. 289173-180 (2011).

15. R.L. Harne and K.W. Wang, J. R. Soc. Interface 
12 104, 20141367 (2015).

16. A. Abolfathi, M.A. Changizi, and M.J. Brennan, J. Phys. Conf. Ser. 744 1, (2016).

17. S.M. Kaplan, Wiley electrical and electronics engineering dictionary (IEEE Press, 2004).

18. A. Thomson and W. Thompson, Acta Biotheor. 26 1, 19-29 (1977).

19. Y.-W. Chin, J.T.-W. Goh, and G.-K. Lau, Insectinspired thoracic mechanism with non-linear stiffness for flapping-wing micro air vehicles, in: 2014 IEEE Int. Conf. Robot. Autom., (IEEE, 2014), pp. 3544-3549. 